\title{
Obesidad materna: severo problema de salud pública en Chile
}

El exceso de peso corporal se ha convertido en un problema de salud pública mayor en todo el mundo y la población obstétrica no escapa a esta epidemia nutricional. La Organización Mundial de la Salud (OMS) define el estado nutricional usando el índice de masa corporal (IMC, calculado como proporción del peso en $\mathrm{kg} / \mathrm{altura}$ en $\mathrm{m} 2$ ). Se considera sobrepeso cuando el IMC está entre 25 y 29,9 y hablamos de obesidad con un IMC de 30 o más (1). Este problema nutricional está asociado al desarrollo de enfermedades crónicas relacionadas con la dieta como diabetes mellitus, infarto agudo al miocardio, accidentes cerebrovasculares, hipertensión y ciertos tipos de cáncer. La mayoría de los países con información nutricional actualizada informan que la mitad o más de su población adulta tiene un IMC $\geq 25$ (2). Según la primera Encuesta Nacional de Salud del 2003 (ENS 2003), Chile se ubicó en la posición número 20 en la clasificación mundial, con un $61 \%$ de los adultos chilenos con IMC en rango de sobrepeso y obesidad (3). Al contrario de los objetivos deseados, este porcentaje de exceso de peso en la población nacional se incrementó a $67 \%$ en la última versión de la encuesta de salud chilena (ENS 2009-2010) (4,5). Especialmente importante para los resultados obstétricos, la encuesta ENS 2009-2010 demostró que el $51 \%$ de las mujeres entre 15 y 44 años de edad tenía un IMC $\geq 25$. En otras palabras, más de la mitad de las mujeres chilenas en edad fértil presenta sobrepeso u obesidad. La importancia de esta creciente proporción de la población obstétrica se debe a las posibles consecuencias de esta alteración nutricional sobre la salud materno-infantil.

\section{Obesidad como el factor de riesgo obstétrico más frecuente}

La obesidad materna (OM) y la excesiva ganancia de peso gestacional (GPG) han sido reconocidas como factores de riesgo independientes para complicaciones maternas y fetales (6). Específicamente en el embarazo, la obesidad se asocia a diferentes morbilidades materno-perinatales, incluyendo malformaciones congénitas, aborto recurrente, diabetes (pre-gestacional y gestacional), hipertensión gestacional y preeclampsia, macrosomia (peso al nacer $>4000 \mathrm{~g}$ ), cesárea, parto instrumental, tromboembolismo, infecciones puerperales, mortalidad materna y mortalidad fetal $(6,7)$. Desde la primera publicación por el Instituto de Medicina (IOM, Estados Unidos de América) en 1990 de las recomendaciones de GPG (8), en EE.UU. se ha producido un aumento del $70 \%$ en la prevalencia de la obesidad antes del embarazo (6). Una situación similar se ha observado en Chile, donde las prevalencias de sobrepeso y OM han aumentado progresivamente desde $18,8 \%$ y $12,9 \%$ en 1987 , a $33 \%$ y el $23 \%$ en 2011 , respectivamente (5). A pesar de que nuestro país ha sido pionero en el desarrollo de guías de GPG que se han adoptado en diversos países vecinos, nuestro sistema de salud no ha logrado revertir la tendencia de aumento progresivo en la incidencia de sobrepeso y OM durante el embarazo. De hecho, aunque el cambio en el uso de la curva de GPG, reemplazando aquella diseñada por Rosso y Mardones (9) por la curva actual de Atalah y cols (10), produjo una reducción significativa en los niveles de OM en el año 2005, la tendencia descrita en la población obstétrica se mantiene intacta en los años siguientes. De cierta forma, es posible que el lento pero continuo aumento de este importante factor de riesgo obstétrico pueda contribuir al estancamiento observado en los indicadores de salud materno-perinatal en los últimos años en nuestro país $(11,12)$. Adicionalmente, la información disponible históricamente en el sistema de salud chileno muestra que la proporción de mujeres que presenta sobrepeso y obesidad a los 6 meses post-parto es aún mayor que durante el embarazo (13), siendo así el proceso reproductivo uno de los factores fundamentales para el incremento de peso de la población femenina en edad reproductiva.

\section{Efecto postnatal de la obesidad materna sobre la descendencia}

Además de las complicaciones perinatales 
asociadas a la obesidad en el embarazo, existe evidencia sobre posibles efectos persistentes y perjudiciales sobre la descendencia. De hecho, la obesidad pregestacional y la GPG excesiva han sido implicadas en un "círculo vicioso transgeneracional de la obesidad". Esto consiste en que embarazadas con sobrepeso u obesidad pueden dar a luz a hijas macrosómicas, que son más propensas a ser obesas durante su propio embarazo posteriormente y a su vez, dar a luz a hijos macrosómicos (14). Se ha reportado que el peso al nacer se asocia directamente con el IMC y el riesgo de obesidad en la adolescencia $(15,16)$. Así, las recomendaciones de GPG deberían tener en cuenta estas influencias de la nutrición materna sobre la salud de la descendencia. Por otro lado, los hijos de mujeres con OM tienen más probabilidades de desarrollar resistencia a la insulina y enfermedades cardiovasculares en el futuro (17). Diversos estudios han evidenciado que la OM tanto pregestacional como gestacional se asocia con la presencia de múltiples factores de riesgo cardiovascular en los hijos. En uno de los estudios más grandes, las mujeres que tuvieron una GPG mayor a las recomendaciones IOM actualizadas el año 2009, fueron más propensas a tener hijos con mayores niveles de IMC, perímetro de cintura, masa grasa, presión arterial sistólica, leptina, proteína C-reactiva e interleucina- 6 y menores niveles de colesterol de alta densidad (HDL) y apolipoproteína A (18). Otros estudios epidemiológicos muestran que la obesidad materna aumenta la incidencia del síndrome metabólico en los niños (19). Estos hallazgos han sido replicados en numerosos estudios similares (20-22). Es destacable que el efecto de la OM sobre la susceptibilidad a la obesidad en la descendencia parece ser independiente de la presencia de diabetes gestacional (DG). Esto debido a que las mujeres con OM, cuyos valores de glicemia son normales durante el embarazo, muestran de igual forma un aumento de la adiposidad en sus hijos (23). El mismo grupo de estudio ha reportado que la obesidad materna está relacionada con un compromiso metabólico ya evidente en el momento del nacimiento, caracterizado por una reducción en la sensibilidad a la insulina y un aumento de marcadores inflamatorios circulantes en el neonato (24). Un análisis más profundo realizado por el grupo español de diabetes y embarazo (GEDE) reportó que la proporción del riesgo materno-perinatal atribuible a la exposición a la OM es mayor al riesgo atribuible a la DG, siendo por ejemplo el $42 \%$ frente al 3,8\% para la macrosomia, el $50 \%$ frente al $9,1 \%$ para la HTA, o el 9,4\% frente al 2,0\% para la predicción de cesárea (25). Así, la OM pregestacional y la GPG excesiva se relacionan de forma independiente con un mayor riesgo de obesidad, resistencia a la insulina y marcadores tempranos de enfermedad cardiovascular en la descendencia, detectables incluso al momento de nacer. Esto es concordante con una revisión epidemiológica reciente que demostró un mayor riesgo de mortalidad por eventos cardiovasculares en los individuos que habían nacido de embarazos con OM, en comparación a aquellos nacidos de embarazos normales desde el punto de vista nutricional (26). Toda esta evidencia desplaza nuestra atención hacia el periodo gestacional como un objetivo de intervención muy importante en la prevención de la epidemia de la obesidad y sus consecuencias asociadas, como la resistencia a la insulina y el riesgo cardiovascular. El adecuado diseño de estrategias preventivas enfocadas en los posibles mecanismos de conexión entre la obesidad materna y el aumento del riesgo de alteraciones nutricionales y metabólicas en la descendencia podría contribuir a reducir el impacto negativo de este problema en la salud de las personas.

Un gran cuerpo de evidencia ha mostrado la contribución diferencial de los factores genéticos y ambientales en el desarrollo de las enfermedades crónicas no transmisibles. En el caso de la obesidad, el cambio demográfico de la población hacia un fenotipo más adiposo en un período relativamente corto, equivalente a sólo una o dos generaciones, argumenta en contra de una contribución genética importante y más bien en favor de mecanismos ambientales y probablemente epigenéticos. De acuerdo con esta mayor relevancia de los factores ambientales, la evidencia reciente sugiere que la prevención de la obesidad en la población debería comenzar incluso antes de la concepción para ser realmente eficaz (27). Para propiciar la reversión de esta tendencia histórica debemos ser capaces de influenciar de forma eficiente en múltiples momentos de interacción con la población femenina en riesgo: antes, durante y después del período gestacional.

\section{Múltiples oportunidades de intervención}

El embarazo es un período crítico de la vida en el que la mujer y la familia en general muestran un nivel de motivación único. Es por esto que es posible evidenciar la completa cesación del consumo de tabaco o alcohol desde el mismo momento en que se conoce la condición de gestante en la gran mayoría de las mujeres. El problema de la OM parte por la gran falta de conciencia respecto de que es efectivamente un problema. Aunque la evidencia es rotunda al mostrar niveles de riesgo elevado para múltiples complicaciones maternas, perinatales, e incluso más allá del embarazo, la población parece reacia a asumir tales riesgos como verdaderos. O bien, no somos capaces de transmitirlo de una forma que permita generar cambios reales. Sin embargo, aún cuando no sea posible convencer a la 
población obstétrica de estos riesgos, estamos obligados a sugerir un control de este factor de la mejor manera posible.

La mejor evidencia disponible al respecto muestra que las intervenciones para optimizar el manejo del peso materno durante el embarazo basadas en la dieta balanceada y el ejercicio controlado son efectivas (28). La intervención dietética con mejores resultados corresponde al régimen calórico balanceado, de bajo contenido en grasa y colesterol, y alto en fibra. La actividad física recomendada involucra ejercicio con entrenamiento de resistencia de baja intensidad. Estas intervenciones son útiles para reducir complicaciones obstétricas como la preeclampsia, hipertensión gestacional y parto prematuro, además de reducir la proporción de neonatos grandes para la edad gestacional y la distocia de hombros (28). No obstante, la gran mayoría de los estudios carece de información específica en relación a la intensidad y duración de las intervenciones evaluadas, los recursos necesarios para su puesta en práctica, el grado de cumplimiento por las pacientes y los factores que pueden facilitar $u$ obstaculizar la aplicación de tales intervenciones. Probablemente sea en estos elementos, relativos a nuestra capacidad como prestadores de salud para la aplicación adecuada de las medidas de control del peso materno con beneficio demostrado, donde aún tenemos una tarea pendiente. Es aquí donde toma relevancia nuestro compromiso y habilidad para coordinar el apoyo del equipo conformado por enfermeras matronas y profesionales de la nutrición y la kinesiología, con el objetivo de optimizar la atención nutricional de nuestra población de embarazadas desde el primer control prenatal.

Sin embargo, la capacidad de modificar el impacto de la OM sobre los resultados materno-perinatales, así como los efectos negativos de esta condición sobre la salud materna e infantil a mediano y largo plazo, no será realmente relevante a menos que logremos fomentar un cambio más integral en la población. Específicamente en el área obstétrica, es imprescindible que el incentivo para el control adecuado del peso materno persista más allá del parto. Esto involucra mantener en primer lugar el apoyo a la lactancia materna exclusiva por al menos 6 meses y podría incluir por ejemplo el diseño de guías claras sobre la mejor forma de propiciar el regreso al peso ideal durante el periodo postparto. Lo que sea que estemos haciendo actualmente como sistema de salud, no ha sido suficiente para lograr este objetivo. Es cierto que la aplicación del reposo postnatal extendido permite actualmente facilitar procesos tan fundamentales como la lactancia materna exclusiva. Sin embargo, no estamos otorgando las oportunidades necesarias para aprovechar este periodo que es ideal para llevar el estado nutricional de la mujer a valores normales.
Es probable que se requiera evaluaciones formales de los posibles impactos de medidas de intervención nutricional y de actividad física durante el período puerperal. Pero no deja de ser interesante el beneficio que estos cambios podrían tener para el resto de los integrantes de la familia, cuyo apoyo es completamente necesario para lograr objetivos que involucran cambios en estilos de vida. En este sentido, el impacto positivo de una adecuada implementación de estas medidas podría sobrepasar con creces el ámbito de la salud femenina en nuestra población.

Finalmente, y no por eso menos importante, resulta muy atingente volver a destacar la gran proporción de mujeres que comienza el embarazo con exceso de peso. Siendo aproximadamente un 51\% de las mujeres en edad fértil, actualmente esta proporción constituye por lejos uno de los factores de riesgo obstétricos más prevalentes y a la luz de las estadísticas actuales, uno de los menos modificables. Es así como el mensaje final debiera ser que nuestros esfuerzos como especialistas dedicados a la salud de la mujer en todas las etapas de la vida, debieran comenzar mucho antes de la gestación. Debemos ser capaces de generar la conciencia necesaria para considerar el exceso de peso materno como un problema prevenible en etapas pregestacionales. En primer lugar, debemos generar conciencia entre nosotros mismos, fomentando la correcta evaluación preconcepcional, que involucre un adecuado consejo y derivación al equipo de apoyo nutricional en caso necesario, previo al intento de embarazo. En segundo lugar, es necesario generar conciencia en el resto de las especialidades y estamentos de salud involucrados en el control de la mujer en etapas previas al embarazo; y por supuesto, debemos generar conciencia en las mujeres mismas, diseñar estrategias que permitan transmitir adecuadamente la información a las futuras embarazadas. Es necesario educar a nuestra población con el objetivo de que sea partícipe de su propio cuidado en salud. La obesidad es habitualmente asociada al desarrollo de enfermedades crónicas a largo plazo, más que a problemas como los descritos en relación al proceso reproductivo. Probablemente al igual que en otros tópicos que involucran modificaciones en estilos de vida como el hábito tabáquico o la protección medio-ambiental, las intervenciones preventivas más eficaces se basan más bien en la educación de nuestros niños.

El sobrepeso y la OM constituyen un problema cuyas consecuencias y estrategias de manejo superan el ámbito obstétrico, afectando en definitiva la salud de la población en general en un marco temporal ampliamente mayor al periodo gestacional mismo. Es por esto que tanto la comunicación de estos riesgos como la implementación de las diferentes estrategias de manejo probablemente 
requieren de un equipo interdisciplinario que vaya más allá del consejo nutricional aislado. Para modificar el impacto actual de este problema en nuestra población, nuestro rol como especialistas en salud de la mujer está inicialmente en contribuir al desarrollo del compromiso necesario tanto a nivel de la comunidad como en los diferentes equipos de atención involucrados.

\section{Dr. Marcelo Farías. PhD. División de Obstetricia y Ginecología. Escuela de Medicina. Pontificia Universidad Católica de Chile.}

\section{REFERENCIAS}

1. Organización Mundial de la Salud, 2003. WHO Technical Report Series № 916: Diet, nutrition and the prevention of chronic diseases. World Health Organization, Geneve. Disponible en: www.who.int/nutrition/ publications/obesity/WHO_TRS_916/en/index.html. Acceso: 12 de octubre de 2013.

2. Organización Mundial de la Salud, 2011. WHO Global Database on Body Mass Index. World Health Organization. Disponible en: apps.who.int/bmi/index.jsp. Acceso: 12 de octubre de 2013.

3. MINSAL, 2003. Encuesta nacional de salud (ENS 2003). Ministerio de Salud, Chile. Disponible en: http:// epi.minsal.cl/epi/html/invest/ens/informefinalens.pdf. Acceso: 10 de octubre de 2013.

4. MINSAL, 2011. Encuesta Nacional de Salud 20092010 (ENS 2009-2010). Ministerio de Salud, Gobierno de Chile, Santiago. Disponible en: http://www.redcronicas.cl/index.php?option=com_docman\&ltemid=314. Acceso: 10 de octubre de 2013.

5. MINSAL, 2011. Indicadores de obesidad en población chilena. Ministerio de Salud, Gobierno de Chile, Santiago. Disponible en: www.redsalud.gov.cl/portal/ url/item/9ac1bf1ed8ee8922e04001011e013229.pdf. Acceso: 10 de octubre de 2013.

6. American College of Obstetrics and Gynecology. ACOG Committee Opinion number 315: Obesity in pregnancy. Obstet Gynecol 2005;106:671-5.

7. Flenady V, Koopmans L, Middleton P, Frøen JF, Smith GC, Gibbons K, et al. Major risk factors for stillbirth in high-income countries: a systematic review and meta-analysis. Lancet 2011;377:1331-40.

8. IOM, 1990. Institute of Medicine, National Academy of Sciences: Nutrition during pregnancy. National Academy Press, Washington, DC.

9. Mardones F, Rosso P. A weight gain chart for pregnant women designed in Chile. Matern Child Nutr 2005;1:77-90.

10. Atalah E, Castillo C, Castro R, Amparo-Aldea P. Propuesta de un nuevo estándar de evaluación nutricional en embarazadas. Rev Med Chil 1997;125:1429-36.

11. Donoso E, Carvajal JA. The change in the epidemiological profile of maternal mortality in Chile will hinder the fulfillment of the Millennium 5th goal]. Rev Med Chil 2012;140:1253-62.

12. Hankins GD, Clark SL, Pacheco LD, OuKeeffe D, DıAlton M, Saade GR. Maternal mortality, near misses, and severe morbidity: lowering rates through designated levels of maternity care. Obstet Gynecol 2012;120(4):929-34.

13. MINSAL, 2013. Evaluación del Programa Nacional de Alimentación Complementaria. Disponible en: www. dipres.gob.cl/595/articles-107673_doc_pdf.pdf. Acceso: 4 de noviembre de 2013.

14. Catalano PM. Obesity and pregnancy--the propagation of a viscous cycle? J Clin Endocrinol Metab 2003;88:3505-6.

15. Curhan GC, Willett WC, Rimm EB, Spiegelman D, Ascherio AL, Stampfer MJ. Birth weight and adult hypertension, diabetes mellitus, and obesity in US men. Circulation 1996;94:3246-50.

16. Oken E, Rifas-Shiman SL, Field AE, Frazier AL, GiIIman MW. Maternal gestational weight gain and offspring weight in adolescence. Obstet Gynecol 2008;112:999-1006.

17. Dörner G, Plagemann A. Perinatal hyperinsulinism as possible predisposing factor for diabetes mellitus, obesity and enhanced cardiovascular risk in later life. Horm Metab Res 1994;26:213-21.

18. Fraser A, Tilling K, Macdonald-Wallis C, Sattar N, Brion MJ, Benfield L, et al. Association of maternal weight gain in pregnancy with offspring obesity and metabolic and vascular traits in childhood. Circulation 2010;121:2557-64.

19. Oken E, Gillman MW. Fetal origins of obesity. Obes Res 2003;11:496-506.

20. Laor A, Stevenson DK, Shemer J, Gale R, Seidman DS. Size at birth, maternal nutritional status in pregnancy, and blood pressure at age 17: population based analysis. BMJ 1997;315:449-53.

21. Clark PM, Atton C, Law CM, Shiell A, Godfrey K, Barker DJ. Weight gain in pregnancy, triceps skinfold thickness, and blood pressure in offspring. Obstet Gynecol 1998;91:103-7.

22. Mamun AA, O'Callaghan M, Callaway L, Williams G, Najman J, Lawlor DA. Associations of gestational weight gain with offspring body mass index and blood pressure at 21 years of age: evidence from a birth cohort study. Circulation 2009;119:1720-7.

23. Sewell MF, Huston-Presley L, Super DM, Catalano P. Increased neonatal fat mass, not lean body mass, is associated with maternal obesity. Am J Obstet Gynecol 2006;195:1100-3.

24. Catalano PM, Presley L, Minium J, Hauguel-de Mouzon S. Fetuses of obese mothers develop insulin resistance in utero. Diabetes Care 2009;32:1076-80.

25. Ricart W, López J, Mozas J, Pericot A, Sancho MA, González N, et al. Body mass index has a greater impact on pregnancy outcomes than gestational hyperglycaemia. Diabetologia 2005;48:1736-42.

26. Reynolds RM, Allan KM, Raja EA, Bhattacharya S, McNeill G, Hannaford PC, et al. Maternal obesity during pregnancy and premature mortality from cardiovascular event in adult offspring: follow-up of 1323 275 person years. BMJ 2013;347:f4539. doi:10.1136/ bmj.f4539.

27. Salsberry PJ, Reagan PB. Dynamics of early childhood overweight. Pediatrics 2005;116:1329-38.

28. Thangaratinam S, Rogozińska $\mathrm{E}$, Jolly $\mathrm{K}$, Glinkowski S, Duda W, Borowiack E, et al. Interventions to reduce or prevent obesity in pregnant women: a systematic review. Health Technol Assess 2012;16 (31):iii-iv, 1-191. doi:10.3310/hta16310 